\title{
MODIFIED METHOD OF KREJNIN AND MURRI FOR THE DETERMINATION OF ABSOLUTE DECLINATIONS
}

\author{
S.A. Tolchelnikova-MuRRI \\ Central Astronomical Observatory USSR Academy of Sciences \\ Pulkovo \\ 196140 Leningrad, USSR
}

\begin{abstract}
Krejnin and Murri's method (1973) enablesenables one to derive absolute declinations of stars in a narrow equatorial zone $|\delta| \leq 10^{\prime}$ from observations near the Earth's equator $|\varphi| \leq 10^{\prime}$. Some systematic effects, including the errors of the value of the micrometer screw for two equatorial instruments (or the scale error if one of the instruments is a PZT), might be determined if a global reduction is used for the original observations from the equator and from those of an astrolabe at latitude $|\varphi| \approx 20^{\circ}$ to $23^{\circ}$. Astrolabes-especially photoelectric ones (Hu 1988) are considered to be the most efficient for determination of absolute declinations of stars and absolute latitudes of the instruments in Tolchel'nikova-Murri (1985).

In $I z v$. GAO No. 206 the method will be published as well as the criterion for estimating the efficiency of different programs, which is required to improve planning in astrometry.
\end{abstract}

\section{References}

Hu Hui, Cai Xing, Wang Rui: 1988, Acta Astron. Sci. 4, 333

Krejnin, E.I., Murri, S.A.: 1973, Astron. zurn., 50, 606

Tolchel'nikova-Murri, S.A.: 1985, "A Method for Determination of Variation of Mean Latitudes and the Secular Polar Motion," Dep. No. 150-185

\section{ON THE DEFINITION OF AN "INERTIAL COORDINATE SYSTEM"}

\author{
S.S. Peruansky \\ Central Astronomical Observatory \\ Pulkovo \\ 196140 Leningrad, USSR
}

ABSTRACT. Astrometry is a branch of science which develops methods for the quantitative descriptions of places and time instants of astronomical events on the basis of observations of celestial bodies. For this purpose a theoretical coordinate system is introduced (e.g. equatorial $\alpha, \delta$ ). The aim of astrometry is to apply this system to the observed reference objects (stars, planets etc.) so that their coordinates $\alpha(t), \delta(t)$ can be calculated according to the relations $\alpha(t)=f_{1}\left(P_{k}, t-t_{0}\right)$ and $\delta(t)=f_{2}\left(P_{\mathrm{k}}, t-t_{\mathrm{o}}\right)$ where $P_{\mathrm{k}}$ are parameters, $t_{\mathrm{o}}$ is the conventional time instant and $t$ is the current time. In order to understand the term inertial coordinate system assume that the coordinates $\alpha\left(t_{\mathrm{i}}\right), \delta\left(t_{\mathrm{i}}\right)$, $i=1,2, \ldots n$ are used for plotting the coordinate origins. If these coincide then the system is conventional-fixed and therefore inertial. Thus, the inertial coordinate system in astrometry is a conventional-fixed reference frame reproduced with the use of celestial bodies whose law of motion is known with sufficient accuracy. 